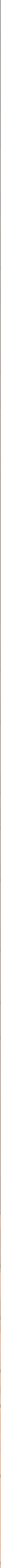




\title{
Multiple period integrals and cohomology
}

\author{
Roelof W. Bruggeman and Youngju Choie
}

\begin{abstract}
We give a version of the Eichler-Shimura isomorphism with a nonabelian $H^{1}$ in group cohomology. Manin has given a map from vectors of cusp forms to a noncommutative cohomology set by means of iterated integrals. We show that Manin's map is injective but far from surjective. By extending Manin's map we are able to construct a bijective map and remarkably this establishes the existence of a nonabelian version of the Eichler-Shimura map.
\end{abstract}

\section{Introduction}

In the theory of modular forms the Eichler-Shimura isomorphism has played an important role, with many applications. For instance, it gives integrality of eigenvalues for Hecke operators and algebraicity of the critical values of the $L$-functions of modular forms, which, for example, enables the construction of $p$-adic $L$-functions and gives a connection to Iwasawa theory as well as the computational aspects of modular form theory. The Eichler-Shimura isomorphism relates spaces of cusp forms of integral weight to a parabolic cohomology group, namely,

$$
S_{k}\left(\mathrm{SL}_{2}(\mathbb{Z})\right) \oplus \bar{S}_{k}\left(\mathrm{SL}_{2}(\mathbb{Z})\right) \cong H_{\mathrm{par}}^{1}\left(\mathrm{SL}_{2}(\mathbb{Z}), \mathbb{C}_{k-2}[X, Y]\right),
$$

where $\mathbb{C}_{k-2}[X, Y]$ is the $\mathrm{SL}_{2}(\mathbb{Z})$-module of homogeneous polynomials of degree $k-2$ in the indeterminates $X, Y$, and where $S_{k}\left(\mathrm{SL}_{2}(\mathbb{Z})\right)\left(\right.$ resp. $\left.\bar{S}_{k}\left(\mathrm{SL}_{2}(\mathbb{Z})\right)\right)$ is the space of holomorphic (resp. antiholomorphic) cusp forms of weight $k$.

The Eichler-Shimura isomorphism was eventually extended in [Knopp 1974] and [Knopp and Mawi 2010] by establishing a canonical isomorphism between 1-cohomology of cofinite discrete subgroups $\Gamma$ of $\mathrm{SL}_{2}(\mathbb{R})$ with appropriate holomorphic coefficients and the space of cusp forms with real weight.

Manin [2005] defined a "nonabelian" $H^{1}$ in group cohomology with values in a nonabelian group, and a map from a product of spaces of cusp forms to this cohomology set, in analogy to the Eichler-Shimura map. Manin's construction uses iterated integrals in the spirit of the multiple zeta values which have proved so useful

Choie is partially supported by NRF-2015049582 and NRF-2013R1A2A2A01068676.

MSC2010: primary 11F67; secondary 11F75.

Keywords: cusp form, iterated integral, noncommutative cohomology. 
in understanding zeta values and mixed Tate motives, for example. Manin's integrals give a way to express multiple $L$-values of modular forms and have been studied by the second author [Choie 2014] and independently in the thesis of N. Provost [2014] recently. In [Choie 2014] the period polynomials whose coefficients are multiple $L$-values were treated as elements in a nonabelian $H^{1}$ for the first time.

In a recent talk at ICM, Brown [2014] mentioned a connection between the iterated integrals of Manin and certain mixed motives. He explained how to interpret motivic multiple zeta values as periods of the pro-unipotent fundamental groupoid of the projective line minus three points $X=\mathbb{P}^{1} \backslash\{0,1, \infty\}$ via iterated integral of smooth 1-forms on a differentiable manifold discussed by Chen [1977]. Hain [2015] discussed the relation between Manin's iterated integrals and the Hodge theory of modular groups. However, it was not clear yet how to relate Manin's iterated integral and Eichler-Shimura theory. Manin's map from spaces of cusp forms to cohomology differs in two aspects from the Eichler-Shimura map: the summand $\bar{S}_{k}\left(\mathrm{SL}_{2}(\mathbb{Z})\right)$ is absent and the map is injective but not surjective.

This paper addresses the second difference by extending Manin's map to more complicated combinations of spaces of cusp forms to obtain a variant of the EichlerShimura isomorphism with values in a nonabelian cohomology $H^{1}$. Our main result (Theorem 6.7) states that there is an extension of Manin's map that is bijective onto a noncommutative cohomology set. It is remarkable that there exists some nonabelian version of the Eichler-Shimura map.

To obtain our main result we modify Manin's construction [2005] in several ways in the spirit of a variant Eichler-Shimura isomorphism established in [Knopp 1974; Knopp and Mawi 2010]: first replace the finite-dimensional spaces of polynomials by spaces of functions on the lower half-plane. Secondly, unlike in the classical Eichler-Shimura isomorphism, antiholomorphic modular forms are not considered. Thirdly, we allow automorphic forms for cofinite discrete subgroups $\Gamma$ of $\mathrm{SL}_{2}(\mathbb{R})$ with arbitrary real weights and multiplier system. Finally, we collapse the number of variables in the iterated integrals.

To be more precise consider the iterated integral

$$
\begin{gathered}
R_{\ell}\left(f_{1}, \ldots, f_{\ell} ; y, x ; t_{1}, \ldots, t_{\ell}\right) \\
:=\int_{\tau_{1}=x}^{y} f_{1}\left(\tau_{1}\right)\left(\tau_{1}-t_{1}\right)^{w_{1}} \int_{\tau_{2}=x}^{\tau_{1}} f_{2}\left(\tau_{2}\right)\left(\tau_{2}-t_{2}\right)^{w_{2}} \\
\quad \ldots \int_{\tau_{\ell}=x}^{\tau_{\ell-1}} f_{\ell}\left(\tau_{\ell}\right)\left(\tau_{\ell}-t_{\ell}\right)^{w_{\ell}} d \tau_{\ell} \cdots d \tau_{2} d \tau_{1},
\end{gathered}
$$

where $x, y$ are in the extended complex upper half-plane and each $t_{j}, 1 \leq j \leq \ell$, is in the lower half-plane. If the $f_{j}$ are cusp forms of even integral weight $w_{j}+2$, the iterated integral defines a polynomial function in the $t_{j}, 1 \leq j \leq \ell$, whose coefficients are multiple $L$-values of $f_{j}$. The resulting iterated integral is holomorphic in 
$\left(t_{1}, \ldots, t_{\ell}\right)$ in the product of $\ell$ copies of the lower half-plane if the $f_{j}$ are cusp forms of real weight. As the order $\ell$ of the iterated integral increases, the relations between iterated integrals become more and more complicated. However, the relations between iterated integrals of order $\ell$ look simple modulo all products of iterated integrals of lower order. Manin $[2005 ; 2006]$ has shown how to give a neat formulation for all relations among iterated integrals of the type indicated in (1-1). His approach works with formal series in noncommuting variables and can be applied to much more general iterated integrals than studied here.

The factors $\left(\tau_{j}-t_{j}\right)^{w_{j}}$ in (1-1) occur also in the definition of cocycles attached to cusp forms. Manin attaches to vectors of cusp forms $\left(f_{1}, \ldots, f_{\ell}\right)$ a cocycle in a noncommutative cohomology set, and thus gives a generalization of the EichlerShimura map. The cohomology has values in a noncommutative subgroup $N(\mathcal{A})$ of the unit group of the noncommutative ring $\mathcal{A}$ of formal power series in noncommuting variables $A_{1}, \ldots, A_{\ell}$ with coefficients in spaces of holomorphic functions on the lower half-plane. The variables $A_{j}$ correspond to spaces of cusp forms $S_{w_{j}+2}\left(\Gamma, v_{j}\right)$ with positive real weights $w_{j}+2$ and corresponding multiplier systems $v_{j}$. Then Manin's approach leads to a map

$$
\prod_{j=1}^{\ell} S_{w_{j}+2}\left(\Gamma, v_{j}\right) \longrightarrow H^{1}(\Gamma ; N(\mathcal{A}))
$$

from a product of finitely many spaces of cusp forms to a noncommutative cohomology set. This (nonlinear) map is far from surjective. In Theorem 6.7 we show that Manin's map can be extended, and that all elements of the cohomology set $H^{1}(\Gamma ; N(\mathcal{A}))$ can be related to combinations of cusp forms by means of iterated integrals. The simplification $t_{1}=\cdots=t_{\ell}$ in the iterated integrals is essential for our methods to work.

Sections 2 and 3 have a preliminary nature. We review the approach of Knopp [1974] of associating cocycles to any cusp form of real weight and the definition of the iterated integrals that we use. Sections 4 and 5 discuss Manin's approach of using formal series in noncommuting variables to associate noncommutative cocycles to vectors of cusp forms. In Section 6 we extend this approach in such a way that the resulting map from collections of cusp forms to noncommutative cohomology is bijective.

\section{Cusp forms and theorem of Knopp and Mawi}

Discrete group. Let $\Gamma$ be a cofinite discrete subgroup of $\mathrm{SL}_{2}(\mathbb{R})$ with translations. Without loss of generality we assume that $\left(\begin{array}{rr}-1 & 0 \\ 0 & -1\end{array}\right) \in \Gamma$. For convenience we conjugate $\Gamma$ into a position for which $\infty$ is among its cusps and such that the subgroup $\Gamma_{\infty}$ of $\Gamma$ fixing $\infty$ is generated by $T=\left(\begin{array}{ll}1 & 1 \\ 0 & 1\end{array}\right)$. 
Notation. For $w \in \mathbb{R}$ and $v$ a corresponding unitary multiplier system, we denote by $S_{w+2}(\Gamma, v)$ the space of holomorphic cusp forms of weight $w+2$ and multiplier system $v$. This is the finite-dimensional space of holomorphic functions $f$ on the upper half-plane satisfying $f(\gamma z)=v(\gamma)(c z+d)^{w+2} f(z)$ for $\gamma \in \Gamma$, with exponential decay upon approach of the cusps. If the weight $w+2$ is integral, a multiplier system is a character.

Functions with at most polynomial growth. By $V(v, w)$, with $w \in \mathbb{R}$ and $v$ a corresponding multiplier system, we denote the space of holomorphic functions on the lower half-plane $\mathfrak{H}^{-}$with at most polynomial growth at the boundary $\mathbb{P}_{\mathbb{R}}^{1}$ of $\mathfrak{H}^{-}$, provided with the action of $\gamma=\left(\begin{array}{ll}a & b \\ c & d\end{array}\right) \in \Gamma$ given by

$$
\left.f\right|_{v,-w} \gamma(t)=v(\gamma)(c t+d)^{-w} f(\gamma t) .
$$

The condition that $f$ has polynomial growth on $\mathfrak{H}^{-}$can be formulated as

$$
|f(t)| \leq C_{1}|t|^{A}+C_{2}|\operatorname{Im} t|^{-A} \quad \text { for all } t \in \mathfrak{H}^{-}, \text {for some } A, C_{1}, C_{2} \geq 0 .
$$

The action $\left.\right|_{v,-w}$ of $\Gamma$ preserves this condition.

Remarks. (a) In [Bruggeman et al. 2014, §1.4] we denoted the representation $V(v, w)$ of $\Gamma$ by $\mathcal{D}_{v,-w}^{-\infty}$ (actually we used $r=w+2$ as the main parameter, and wrote $\left.\mathcal{D}_{v, 2-r}^{-\infty}\right)$.

(b) The polynomial growth condition in (2-2) can be formulated in terms of an estimate by one function $Q(t)=|\operatorname{Im} t| /|t-i|^{2}$ as $|f(t)| \leq C Q(t)^{-A}$ for some $A, C \geq 0$. See the discussion in [Bruggeman et al. 2014, §1.5].

Knopp's cocycles associated to cusp forms. Knopp [1974] associated to cusp forms $f \in S_{w+2}(\Gamma, v)$ a cocycle $\bar{\psi}_{f}$ given by

$$
\bar{\psi}_{f, \gamma}(z)=\overline{\int_{\gamma^{-1} \infty}^{\infty} f(\tau)(\tau-\bar{z})^{w} d \tau} .
$$

This cocycle takes values in the holomorphic functions on the upper half-plane $\mathfrak{H}$ that have at most polynomial growth on $\mathfrak{H}$ in the sense of (2-2) (now with $t$ replaced by $z \in \mathfrak{H}$ ). We avoid the complex conjugation by taking a cocycle with values in the holomorphic functions on the lower half-plane $\mathfrak{H}^{-}$with at most polynomial growth at the boundary:

$$
\psi_{f, \gamma}(t)=\int_{\tau=\gamma^{-1} \infty}^{\infty} f(\tau)(\tau-t)^{w} d \tau .
$$

So $\psi_{f}$ has values in the $\Gamma$-module $V(v, w)$. 
Theorem 2.1 [Knopp and Mawi 2010]. For real weight $w+2$ and corresponding unitary multiplier system $v$, the map $f \mapsto\left[\psi_{f}\right]$ determines a linear bijection

$$
S_{w+2}(\Gamma, v) \longrightarrow H^{1}(\Gamma ; V(v, w)) .
$$

Knopp [1974] conjectured this result, and proved it for many cases. Finally, the remaining cases were completed in [Knopp and Mawi 2010].

Remarks. (a) A multiplier system $v$ is called unitary if $|v(\gamma)|=1$ for all $\gamma \in \Gamma$.

(b) Since $S_{w+2}(\Gamma, v)=\{0\}$ for $w+2 \leq 0$, the theorem implies that the cohomology groups vanish as well for $w+2 \leq 0$.

(c) If $w \in \mathbb{Z}_{\geq 0}$, the cocycles take values in polynomial functions on $\mathfrak{H}^{-}$, which for the trivial multiplier system form a submodule of $V(1, w)$ isomorphic to $\mathbb{C}_{w}[X, Y]$.

If the multiplier system $v$ has values only in $\{1,-1\}$ then conjugation gives cocycles in the same module. The Eichler-Shimura theory gives the parabolic cohomology group with values in polynomial functions of degree at most $w$ as the direct sum of the images of the two maps $f \mapsto\left[\psi_{f}\right]$ and $f \mapsto\left[\bar{\psi}_{f}\right]$. However, in the large module of polynomially growing functions, the cocycles $\bar{\psi}_{f}$ become coboundaries. Also the cocycles associated to Eisenstein series become coboundaries over the module of functions with at most polynomial growth.

(d) Knopp [1974] shows that the parabolic cohomology group $H_{\mathrm{par}}^{1}(\Gamma ; V(v, w))$ is equal to the cohomology group $H^{1}(\Gamma ; V(v, w))$.

\section{Iterated integrals}

By taking $t_{1}=\cdots=t_{\ell}=t$ we consider the following holomorphic function in $t$ running through the lower half-plane:

$$
\begin{array}{r}
R_{\ell}\left(f_{1}, \ldots, f_{\ell} ; y, x ; t\right):=\int_{\tau_{1}=x}^{y} f_{1}\left(\tau_{1}\right)\left(\tau_{1}-t\right)^{w_{1}} \int_{\tau_{2}=x}^{\tau_{1}} f_{2}\left(\tau_{2}\right)\left(\tau_{2}-t\right)^{w_{2}} \\
\cdots \int_{\tau_{\ell}=x}^{\tau_{\ell-1}} f_{\ell}\left(\tau_{\ell}\right)\left(\tau_{\ell}-t\right)^{w_{\ell}} d \tau_{\ell} \cdots d \tau_{2} d \tau_{1} .
\end{array}
$$

It is a multilinear form on $\prod_{j=1}^{\ell} S_{w_{j}+2}\left(\Gamma, v_{j}\right)$ for $\ell$ pairs $\left(v_{1}, w_{1}\right), \ldots,\left(v_{\ell}, w_{\ell}\right)$ of real numbers $w_{j}$ and corresponding unitary multiplier systems $v_{j}$. The parameter $t$ is in the lower half-plane $\mathfrak{H}^{-}$. The value of the iterated integral does not depend on the path of integration, provided we take care to approach cusps along geodesic half-lines (for instance, vertically).

The most interesting case is $y=\gamma^{-1} \infty, \gamma \in \Gamma$, and $x=\infty$. For $\ell=1$ this gives the value $\psi_{f_{1}, \gamma}$ of the cocycle in (2-3). That is why we call $R_{\ell}\left(f_{1}, \ldots, f_{\ell} ; \gamma^{-1} \infty, \infty ; t\right)$ a multiple period integral. 
Functions with at most polynomial growth. The condition of polynomial growth in (2-2) is preserved by the action of $\Gamma$ given for $\gamma=\left(\begin{array}{ll}a & b \\ c & d\end{array}\right)$ by

$$
\begin{aligned}
\left.h\right|_{\boldsymbol{v},-\boldsymbol{w}} \gamma(t) & =\boldsymbol{v}(\gamma)^{-1}(c t+d)^{\boldsymbol{w}} h(\gamma t), \\
\boldsymbol{v}(\gamma) & =v_{1}(\gamma) v_{2}(\gamma) \cdots v_{\ell}(\gamma), \\
\boldsymbol{w} & =w_{1}+w_{2}+\cdots+w_{\ell} .
\end{aligned}
$$

By $V(\boldsymbol{v}, \boldsymbol{w})$ we denote the vector space of holomorphic functions on $\mathfrak{H}^{-}$with the action $\left.\right|_{v,-w}$ given in (3-2). Multiplication of functions gives a bilinear map $V(\boldsymbol{v} ; \boldsymbol{w}) \times V\left(\boldsymbol{v}^{\prime} ; \boldsymbol{w}^{\prime}\right) \rightarrow V\left(\boldsymbol{v} \boldsymbol{v}^{\prime} ; \boldsymbol{w}+\boldsymbol{w}^{\prime}\right)$. The action behaves according to the rule

$$
\left(\left.h\right|_{\boldsymbol{v},-\boldsymbol{w}} \gamma\right)\left(\left.h^{\prime}\right|_{\boldsymbol{v}^{\prime},-\boldsymbol{w}^{\prime}} \gamma\right)=\left.\left(h h^{\prime}\right)\right|_{\boldsymbol{v} \boldsymbol{v}^{\prime},-\boldsymbol{w}-\boldsymbol{w}^{\prime}} \gamma .
$$

Lemma 3.1. For $\boldsymbol{f}=\left(f_{1}, \ldots, f_{\ell}\right) \in \prod_{j=1}^{\ell} S_{w_{j}+2}\left(\Gamma, v_{j}\right)$, the multiple period integral $R_{\ell}(f ; y, x ; \cdot)$ defines an element of $V(\boldsymbol{v}, \boldsymbol{w})$.

Proof. Each cusp form has at most polynomial growth on $\mathfrak{H}$, and has exponential decay at cusps when the cusp is approached along a geodesic half-line. This implies that the iterated integral in (3-1) has at most polynomial growth in $t$ and $\tau_{\ell-1}$. Successively this also implies polynomial growth in $\tau_{j-1}$ and $t$ of the further integrals.

Trivial relation. Directly from the definition we have

$$
R_{\ell}(f ; x, x ; t)=0 .
$$

Lemma 3.2. For $\gamma \in \Gamma$,

$$
R_{\ell}\left(f ; \gamma^{-1} y, \gamma^{-1} x ; t\right)=\left.R_{\ell}(f ; y, x ; \cdot)\right|_{v,-w} \gamma(t) .
$$

Proof. In the following computation all $\tau_{j}$ are replaced by $\gamma \tau_{j}$, with $\gamma=\left(\begin{array}{ll}a & b \\ c & d\end{array}\right) \in \Gamma$ :

$$
\begin{aligned}
&\left.R_{\ell}(\boldsymbol{f} ; x, y ; \cdot)\right|_{\boldsymbol{v},-w} \gamma(t) \prod_{j=1}^{\ell}\left(v_{j}(\gamma)^{-1}(c t+d)^{w_{j}}\right) \int_{\tau_{1}=x}^{y} f_{1}\left(\tau_{1}\right)\left(\tau_{1}-\gamma t\right)^{w_{1}} \int_{\tau_{\ell}=x}^{\tau_{\ell-1}} f_{\ell}\left(\tau_{\ell}\right)\left(\tau_{\ell}-\gamma t\right)^{w_{\ell}} \\
& \quad=\prod_{j=1}^{\ell}\left(v_{j}(\gamma)^{-1}(c t+d)^{w_{j}}\right) \int_{\tau_{1}=\gamma^{-1} x}^{\gamma^{-1} y} f_{1}\left(\gamma \tau_{1}\right) \frac{\left(\tau_{1}-t\right)^{w_{1}}}{\left(c \tau_{1}+d\right)^{w_{1}}(c t+d)^{w_{1}}} \\
& \quad \int_{\tau_{\ell}=\gamma^{-1} x}^{\gamma^{-1}\left(\gamma \tau_{\ell-1}\right)} f_{\ell}\left(\gamma \tau_{\ell}\right) \frac{\left(\tau_{\ell}-t\right)^{w_{\ell}}}{\left(c \tau_{\ell}+d\right)^{w_{\ell}}(c t+d)^{w_{\ell}}} \frac{d \tau_{\ell}}{\left(c \tau_{\ell}+d\right)^{2}} \cdots \frac{d \tau_{1}}{\left(c \tau_{1}+d\right)^{2}} \\
&=R_{\ell}\left(\boldsymbol{f} ; \gamma^{-1} y, \gamma^{-1} x ; t\right) .
\end{aligned}
$$

Cocycles. For $\ell=1$ we get the cocycle $\psi_{f}$ in (2-3):

$$
\psi_{f, \gamma}(t)=-R_{1}\left(f ; \gamma^{-1} \infty, \infty ; t\right) .
$$


Decomposition. It is easy to see that the cocycles in (2-3) satisfy the cocycle relation

$$
c_{\gamma \delta}=c_{\gamma} \mid \delta+c_{\delta}
$$

for $\gamma, \delta \in \Gamma$ : use the decomposition relation $\int_{b}^{a}+\int_{c}^{b}=\int_{c}^{a}$ for integrals together with the invariance relation in Lemma 3.2.

There are decomposition relations for the iterated integrals in (3-1), which can be obtained by application of the decomposition relation for integrals of one variable to the subintegrals in (3-1). For the orders 2 and 3 these relations take the form

$$
\begin{aligned}
& R_{2}\left(f_{1}, f_{2} ; z, y ; t\right)+R_{2}\left(f_{1}, f_{2} ; y, x ; t\right)-R_{2}\left(f_{1}, f_{2} ; z, x ; t\right) \\
& \quad=R_{1}\left(f_{1} ; z, y ; t\right) R_{1}\left(f_{2} ; y, x ; t\right), \\
& R_{3}\left(f_{1}, f_{2}, f_{3} ; z, y ; t\right)+R_{3}\left(f_{1}, f_{2}, f_{3} ; y, x ; t\right)-R_{3}\left(f_{1}, f_{2}, f_{2} ; z, x ; t\right) \\
& \quad=-R_{1}\left(f_{1} ; z, y ; t\right) R_{2}\left(f_{2}, f_{3} ; y, x ; t\right)+R_{2}\left(f_{1}, f_{2} ; z, y ; t\right) R_{1}\left(f_{3} ; y, x ; t\right) .
\end{aligned}
$$

We have written these relations in such a way that the quantity on the left should be zero if the standard decomposition would hold. On the right is a correction term consisting of products of iterated integrals of lower order.

Example. The decomposition relations can be used to obtain relations between values of multiple $L$-functions at special points, as studied in [Choie 2014] and in the thesis by Provost [2014] independently.

Let us take $\Gamma=\mathrm{SL}_{2}(\mathbb{Z})$, and assume that $v_{1}=v_{2}=1$, and $w_{1}, w_{2} \in 2 \mathbb{Z}_{\geq 0}$. This implies that the multiple integrals yield polynomial functions in the variable $t$. We apply (3-7) with $z=x=\infty$ and $y=0$. With (3-4),

$$
R_{2}\left(f_{1}, f_{2} ; \infty, 0 ; t\right)+R_{2}\left(f_{1}, f_{2} ; 0, \infty ; t\right)=R_{1}\left(f_{1} ; \infty, 0 ; t\right) R_{1}\left(f_{2} ; 0, \infty ; t\right) .
$$

Using the binomial theorem, we see that $R_{1}(f ; \infty, 0 ; t)$ is a polynomial in $t$ with coefficients that can be expressed in values of completed $L$-functions. In a similar way, $R_{2}\left(f_{1}, f_{2} ; \infty, 0 ; t\right)$ is a polynomial in $t$ with coefficients that can be expressed in values of a completed multiple $L$-function of order 2 as defined in [Choie 2014, (2.6)]. With Lemma 3.2,

$$
R_{2}\left(f_{1}, f_{2} ; 0, \infty ; \cdot\right)=\left.R_{2}\left(f_{1}, f_{2} ; \infty, 0 ; \cdot\right)\right|_{-w} S,
$$

where $S=\left(\begin{array}{rr}0 & -1 \\ 1 & 0\end{array}\right)$. In this way, the decomposition relation (3-7) implies the equality of two polynomials. Comparing coefficients leads to the relation in [Choie 2014, Theorem 3.1].

This account is a simplification. The decomposition relations are valid for the iterated integrals in (1-1), and lead for $w_{j} \in 2 \mathbb{Z}_{\geq 0}$ to polynomials in two variables. Choie [2014] works in that generality. 


\section{Formal series}

Manin [2005; 2006] has indicated a way to give structure to the decomposition relations of any order. His approach works in a general context of iterated integrals associated to cusp forms. The factors $\left(\tau_{j}-t\right)^{w}$ of the kernel in (3-1) and $\left(\tau_{j}-t_{j}\right)$ in (1-1) may be replaced by more general factors, for instance, by factors leading to iterated $L$-integrals as studied in [Choie 2014]. Here we use Manin's formalism for the iterated integrals in (3-1).

We keep fixed $\ell$ combinations of a weight $w_{j}+2 \in \mathbb{R}$ and a corresponding unitary multiplier system $v_{j}$. For a vector $\boldsymbol{f}=\left(f_{1}, \ldots, f_{\ell}\right) \in \prod_{j=1}^{\ell} S_{w_{j}+2}\left(\Gamma, v_{j}\right)$ of length $\ell$, we form iterated integrals of arbitrary order

$$
R_{n}\left(f_{m_{1}}, f_{m_{2}}, \ldots, f_{m_{n}} ; y, x ; t\right)
$$

for any choice $m=\left(m_{1}, \ldots, m_{n}\right) \in\{1, \ldots, \ell\}^{n}$, for any $n \geq 0$. For $n=0$ we define this quantity to be 1 . The same $f_{j}$ may occur several times as $f_{m_{i}}$. So we do not get linearity in $f_{j}$. The result is a holomorphic function on $\mathfrak{H}^{-}$, and has at most polynomial growth by Lemma 3.1 .

To formulate the $\Gamma$-equivariance, we put for $m=\left(m_{1}, m_{2}, \ldots, m_{n}\right)$

$$
\boldsymbol{v}(m):=v_{m_{1}} v_{m_{2}} \cdots v_{m_{n}}, \quad \boldsymbol{w}(m):=w_{m_{1}}+w_{m_{2}}+\cdots+w_{m_{n}} .
$$

We consider the iterated integral in (4-1) as an element of $V(\boldsymbol{v}(m), \boldsymbol{w}(m))$. For the empty sequence $m=()$ we put $V(\boldsymbol{v}(), \boldsymbol{w}())=\mathbb{C}$ with the trivial action $\left.\right|_{1,0}$. Multiplication follows the rule in (3-3). Lemma 3.2 can be applied.

Power series in noncommuting variables. We choose $\ell$ spaces of cusp forms $S_{w_{j}+2}\left(\Gamma, v_{j}\right)$ with $w_{j}+2>0$ and unitary multiplier systems $v_{j}$, for $1 \leq j \leq \ell$. We indicate this choice by the symbol $\mathcal{A}$. For this choice $\mathcal{A}$ we take $\ell$ noncommuting variables $A_{1}, A_{2}, \ldots, A_{\ell}$.

Let $\boldsymbol{O}(\mathcal{A})$ be the set of formal power series in the $A_{j}$ for which the coefficient of the monomial $A_{m_{1}} A_{m_{2}} \cdots A_{m_{n}}$ is in $V(\boldsymbol{v}(m), \boldsymbol{w}(m))$ for each $m \in\{1, \ldots, \ell\}^{n}$. The constant term is in $V(\boldsymbol{v}(), \boldsymbol{w}())=\mathbb{C}$. The relation (3-3) implies that $\boldsymbol{O}(\mathcal{A})$ is a ring.

Formal series associated to vectors of cusp forms. Following Manin we combine all iterated integrals in (4-1) as coefficients of an element of the ring $\boldsymbol{O}(\mathcal{A})$. Let

$$
S_{\mathcal{A}}(\Gamma)=\prod_{j=1}^{\ell} S_{w_{j}+2}\left(\Gamma, v_{j}\right) .
$$

For $\boldsymbol{f}=\left(f_{1}, \ldots, f_{\ell}\right) \in S_{\mathcal{A}}(\Gamma)$ define the formal series $J(\boldsymbol{f} ; y, x ; t) \in \boldsymbol{O}(\mathcal{A})$ by $J(f ; y, x ; t)$

$$
=1+\sum_{n \geq 1} \sum_{m_{1}, \ldots, m_{n} \in\{1, \ldots, \ell\}} R_{n}\left(f_{m_{1}}, f_{m_{2}}, \ldots, f_{m_{n}} ; y, x ; t\right) A_{m_{1}} A_{m_{2}} \cdots A_{m_{n}} .
$$


Remarks. (a) $J(f ; z, w ; \cdot)$ is an invertible element of $\boldsymbol{O}(\mathcal{A})$ since it has a nonzero constant term.

(b) The coefficients $R_{n}\left(f_{m_{1}}, \ldots, f_{m_{n}} ; y, x ; t\right)$ are continuous functions of $y, x \in \mathfrak{H}^{*}$, and are holomorphic in $x, y \in \mathfrak{H}$.

(c) The $A_{j}$ codes for the space $S_{w_{j}+2}\left(\Gamma, v_{j}\right)$. This approach differs from that in [Manin 2005, §2]. There the formal variables code for linearly independent elements of the space $\prod_{j} S_{w_{j}+2}\left(\Gamma, v_{j}\right)$.

Action of $\Gamma$. We define an action of $\Gamma$ on $\boldsymbol{O}(\mathcal{A})$ by the action $\left.\right|_{\boldsymbol{v}(m),-\boldsymbol{w}(m)}$ on the coefficient of $A_{m_{1}} \cdots A_{m_{n}}$. Lemma 3.2 implies the relation

$$
J\left(f ; \gamma^{-1} y, \gamma^{-1} x ; \cdot\right)=J(f ; y, x ; \cdot) \mid \gamma \quad \text { for each } \gamma \in \Gamma .
$$

Multiplication properties. These formal series satisfy for $z, y, x \in \mathfrak{H}^{*}$

$$
\begin{aligned}
& J(f ; x, x ; t)=1, \\
& J(f ; x, y ; t)=J(f ; y, x ; t)^{-1}, \\
& J(f ; z, x ; t)=J(f ; z, y ; t) J(f ; y, x ; t) .
\end{aligned}
$$

We will prove a more general result in Proposition 6.3.

These relations encapsulate infinitely many relations between multiple period integrals. The reader who takes the trouble to compare the coefficients of $A_{1} A_{2}$ in (4-8) obtains the relation (3-7). Similarly, relation (3-8) is given by the coefficient of $A_{1} A_{2} A_{3}$.

Commutative example. In the modular case we may look at $w=N / 2-2$ for some $N \in \mathbb{Z}_{\geq 1}$. As the corresponding multiplier system we choose $v_{N / 2}$ determined by

$$
v_{N / 2}\left(\begin{array}{ll}
1 & 1 \\
0 & 1
\end{array}\right)=e^{\pi i N / 12}, \quad v_{N / 2}\left(\begin{array}{rr}
0 & -1 \\
1 & 0
\end{array}\right)=e^{-\pi i N / 4} .
$$

For $1 \leq N \leq 24$ the space of cusp forms is one-dimensional, in fact:

$$
S_{N / 2+2}\left(\Gamma(1), v_{N / 2}\right)=\mathbb{C} \eta^{N},
$$

where $\eta(\tau)=q^{1 / 24} \prod_{n \geq 1}\left(1-q^{n}\right), q=e^{2 \pi i \tau}$, is the Dedekind eta function.

We take $\ell=1$, with $w_{1}=N / 2-2$ and multiplier system $v_{N / 2}$. The $\operatorname{ring} \boldsymbol{O}(\mathcal{A})$ is a commutative ring of formal power series in one variable $A$. The coefficient of $A^{m}$ is in the $\Gamma(1)$-module $V\left(v_{m N / 2}, m N / 2-2 m\right)$.

If we take $1 \leq N \leq 24$, then with $\boldsymbol{f}=\left(\eta^{N}\right)$ we get in (4-4)

$$
J(f ; y, x ; t)=1+\sum_{n \geq 1} R_{n}\left(\left(\eta^{N}\right)^{\times n} ; y, x ; t\right) A^{n},
$$

where $\left(\eta^{N}\right)^{\times n}$ means a sequence of $n$ copies of $\eta^{N}$.

If $N>24$ we still can work with $\boldsymbol{f}=(f)$, but now $f$ need not be a multiple of $\eta^{N}$. 


\section{From cusp forms to noncommutative cohomology}

Manin uses relation (4-8) to associate a noncommutative cocycle to the vector $\boldsymbol{f}=\left(f_{1}, \ldots, f_{\ell}\right)$ of cusp forms. We first reformulate Manin's description [2005, $\S 1]$ of noncommutative cohomology for a right action, and then determine the map from vectors of cusp forms to noncommutative cohomology.

Noncommutative cohomology. Let $G$ and $N$ be groups, written multiplicatively, and suppose that for each $g \in G$ there is an automorphism $n \mapsto n \mid g$ of $N$ such that the map $g \mapsto \mid g$ is an antihomomorphism from $G$ to the automorphism group $\operatorname{Aut}(N)$, i.e., $n|(g h)=(n \mid g)| h$ for $n \in N$ and $g, h \in G$.

A map $\rho: G \rightarrow N$ is called a 1-cocycle if it satisfies

$$
\rho_{g h}=\left(\rho_{g} \mid h\right) \rho_{h} \quad \text { for all } g, h \in G .
$$

The set of such cocycles is called $Z^{1}(G ; N)$. It is not a group. Nevertheless it contains the special element $1: g \mapsto 1$.

The group $N$ acts on $Z^{1}(G ; N)$ from the left, by $\rho \mapsto{ }^{n} \rho$ defined by

$$
{ }^{n} \rho_{g}=(n \mid g) \rho_{g} n^{-1} .
$$

The cohomology set $H^{1}(G ; N)$ is the set of $N$-orbits in $Z^{1}(G ; N)$ for this action. The orbit of the cocycle $g \mapsto 1$ is called the set of coboundaries $B^{1}(G ; N)$.

Noncommutative cocycles attached to a sequence of cusp forms. As the group $N$ we use the subgroup $N(\mathcal{A})$ of the group of those units in $\boldsymbol{O}(\mathcal{A})^{*}$ that have constant term equal to 1 . The series $J(f ; y, x ; \cdot)$ in (4-4) is an element of $N(\mathcal{A})$.

Following Manin we define for $\boldsymbol{f}=\left(f_{1}, \ldots, f_{\ell}\right) \in S_{\mathcal{A}}(\Gamma)$ and $x \in \mathfrak{H}^{*}$

$$
\Psi(\boldsymbol{f})_{\gamma}^{x}(t)=J\left(\boldsymbol{f} ; \gamma^{-1} x, x ; t\right) .
$$

The properties (4-5) and (4-8) imply that this defines a noncommutative cocycle $\Psi(f)^{x} \in Z^{1}(\Gamma ; N(\mathcal{A}))$, and that its cohomology class $\operatorname{Coh}_{\mathcal{A}}(f) \in H^{1}(\Gamma ; N(\mathcal{A}))$ does not depend on the choice of the base-point $x$. We write $\Psi(f)=\Psi(f)^{\infty}$.

Proposition 5.1. The map

$$
\mathrm{Coh}_{\mathcal{A}}: S_{\mathcal{A}}(\Gamma) \rightarrow H^{1}(\Gamma ; N(\mathcal{A}))
$$

is injective.

Proof. Suppose that the cocycles $\Psi\left(f_{1}, \ldots, f_{\ell}\right)$ and $\Psi\left(f_{1}^{\prime}, \ldots, f_{\ell}^{\prime}\right)$ are in the same cohomology class. Then there is an $n \in N(\mathcal{A})$ such that for all $\gamma \in \Gamma$

$$
\Psi\left(f_{1}^{\prime}, \ldots, f_{\ell}^{\prime}\right)_{\gamma}=(n \mid \gamma) \Psi\left(f_{1}, \ldots, f_{\ell}\right)_{\gamma} n^{-1} .
$$


We denote the coefficient of $A_{j}$ in $n$ by $n_{j} \in V\left(v_{j}, w_{j}\right)$. In relation (5-5) we consider only the constant term and the term with $A_{j}$, and work modulo all other terms:

$$
1-\psi_{f_{j}^{\prime}, \gamma} A_{j} \equiv\left(1+n_{j} A_{j}\right)\left(1-\psi_{f_{j}, \gamma} A_{j}\right)\left(1-n_{j} A_{j}\right) .
$$

Taking the factor of $A_{j}$ gives

$$
-\psi_{f_{j}^{\prime}, \gamma}=\left.n_{j}\right|_{v_{j},-w_{j}} \gamma-\psi_{f_{j}, \gamma}-n_{j} .
$$

In other words, $\psi_{f_{j}^{\prime}}$ and $\psi_{f_{j}}$ differ by a coboundary. We have used the noncommutative relation (5-5) in $N(\mathcal{A})$ to get a commutative relation in $V\left(v_{j}, w_{j}\right)$.

By the theorem of Knopp and Mawi (Theorem 2.1) we conclude that $f_{j}^{\prime}=f_{j}$ for all $j$. Hence $\mathrm{Coh}_{\mathcal{A}}$ is injective.

Remarks. (a) Implicit in the proof is the quotient of $\mathcal{A}$ by the ideal generated by all monomials in the $A_{j}$ with degree 2 . The corresponding quotient of $N(\mathcal{A})$ is isomorphic to the direct sum of the $V\left(v_{j}, w_{j}\right)$.

(b) The injectivity of the map from cusp forms to cocycles is a point in common for this result, the theorem of Knopp and Mawi, and the classical Eichler-Shimura result. The bijectivity in the theorem of Knopp and Mawi is not shared by the classical result, where conjugates of cocycles also determine cohomology classes. In the next section we will see that the whole group $H^{1}(\Gamma ; N(\mathcal{A}))$ can be described with cusp forms, but in a more complicated way than by the map $\operatorname{Coh}_{\mathcal{A}}$.

Commutative example. Toward the end of page 653 we considered the case $\ell=1$. Then $N(\mathcal{A})$ is a commutative group, and $H^{1}(\Gamma ; N(\mathcal{A})$ ) is a cohomology group.

When $\Gamma=\Gamma(1)$, with the choices and notations indicated on page 653 , the cocycle $\Psi\left(\eta^{N}\right)$ vanishes on $\left(\begin{array}{ll}1 & 1 \\ 0 & 1\end{array}\right)$ (hence may be called a parabolic cocycle), and is determined by its value on $S=\left(\begin{array}{cc}0 & -1 \\ 1 & 0\end{array}\right)$ :

$$
\Psi\left(\eta^{N}\right)_{S}(t)=J\left(\eta^{N} ; 0, \infty ; t\right)=1+\sum_{n \geq 1} R_{n}\left(\left(\eta^{N}\right)^{\times n} ; 0, \infty ; t\right) A^{n} .
$$

The coefficient of $A^{n}$ is an iterated period integral of $\eta^{N}$. The cocycle satisfies the well known relations $\left(\Psi\left(\eta^{N}\right)_{S} \mid S\right) \Psi\left(\eta^{N}\right)_{S}=1$ and $\Psi\left(\eta^{N}\right)_{S}=\Psi\left(\eta^{N}\right)_{S}\left|T^{\prime} \Psi\left(\eta^{N}\right)_{S}\right| T$, with $T^{\prime}=\left(\begin{array}{ll}1 & 0 \\ 1 & 1\end{array}\right)=T S T$.

\section{Noncommutative cocycles and collections of cusp forms}

The proof of Proposition 5.1 is based on the fact that the vector of cusp forms $f$ can be recovered from the terms of degree 1 in the formal series $J\left(f ; \gamma^{-1} \infty, \infty ; t\right)$. In this section we associate to collections of cusp forms noncommutative cocycles of a more general nature. 
We keep fixed the choice $\mathcal{A}$ of positive weights $w_{1}+2, \ldots, w_{\ell}+2$ and corresponding multiplier systems $v_{1}, \ldots, v_{\ell}$. To each monomial $B=A_{m_{1}} \cdots A_{m_{d}}$ in $\boldsymbol{O}(\mathcal{A})$ we associate the shifted weight $\boldsymbol{w}(B):=\boldsymbol{w}(m)$ and the multiplier system $\boldsymbol{v}(B):=\boldsymbol{v}(m)$ as defined in (4-2) for $m=\left(m_{1}, \ldots, m_{d}\right) \in\{1, \ldots, \ell\}^{d}$. So $\boldsymbol{w}(B)$ and $\boldsymbol{v}(B)$ depend only on the factors $A_{m_{i}}$ occurring in $B$, not on their order.

Definition 6.1. We call the degree $d(B)$ of the monomial $B=A_{m_{1}} \cdots A_{m_{d}}$ the number $d$ of factors $A_{j}(1 \leq j \leq \ell)$ occurring in it.

Let $\mathcal{B}(\mathcal{A})$ be the set of all monomials $B$ in $A_{1}, \ldots, A_{\ell}$ with $d(B) \geq 1$ for which $S_{\boldsymbol{w}(B)+2}(\Gamma, \boldsymbol{v}(B)) \neq\{0\}$. We put

$$
S(\mathcal{A} ; \Gamma):=\prod_{B \in \mathcal{B}(\mathcal{A})} S_{\boldsymbol{w}(B)+2}(\Gamma, \boldsymbol{v}(B)) .
$$

Remarks. (a) The space of cusp forms $S_{\boldsymbol{w}(B)+2}(\Gamma, \boldsymbol{v}(B))$ may be zero. In fact, this is necessarily the case if $\boldsymbol{w}(B) \leq-2$. For $\boldsymbol{w}(B)>-2$ it may also happen to be zero, depending on $\Gamma$ and $\boldsymbol{v}(B)$.

(b) The set $\mathcal{B}(\mathcal{A})$ is often infinite. We recall that elements of infinite direct sums of vector spaces have zero components at all but finitely many $B \in \mathcal{B}(\mathcal{A})$. Here we use the product. Its elements may have nonzero components for all $B$.

(c) We denote elements of $S(\mathcal{A} ; \Gamma)$ by $\boldsymbol{h}$, with component $\boldsymbol{h}(B)$ in the factor corresponding to the monomial $B$.

(d) There may be more than one monomial $B$ for which $S_{w(B)+2}(\Gamma, v(B))$ is equal to a given space of cusp forms. See (f2) below for an example where this happens for infinitely many monomials.

(e) The space $S_{\mathcal{A}}(\Gamma)=\prod_{j=1}^{\ell} S_{w_{j}+2}\left(\Gamma, v_{j}\right)$ in (4-3) may be considered as a subspace of $S(\mathcal{A} ; \Gamma)$. To do this we define for a given $\boldsymbol{f}=\left(f_{1}, \ldots, f_{\ell}\right) \in S_{\mathcal{A}}(\Gamma)$ the element $\boldsymbol{h} \in S(\mathcal{A} ; \Gamma)$ by

$$
\boldsymbol{h}\left(A_{j}\right)=f_{j}, \quad \boldsymbol{h}(B)=0, \quad \text { if } d(B) \geq 2 .
$$

(f) In the commutative case $\ell=1$ we have $\mathcal{B}(\mathcal{A}) \subset\left\{A^{n}: n \in \mathbb{Z}_{\geq 1}\right\}$. We consider three specializations of the example on page 653 .

(f1) Take $N=24$. So $\mathcal{B}(\mathcal{A})=\left\{A^{n}: n \in \mathbb{Z}_{\geq 1}\right\}, \boldsymbol{w}(B)=10 n$ and $\boldsymbol{v}(B)=v_{12}=v_{0}=1$. Hence

$$
S(\mathcal{A} ; \Gamma(1))=\prod_{n \geq 1} S_{10 n+2}(\Gamma(1), 1) .
$$

(f2) Take $N=4$. So $\boldsymbol{w}\left(A^{n}\right)=0$ for all $n \geq 1$ and the space $S_{2}\left(\Gamma(1), v_{2 n}\right)$ is equal to $\mathbb{C} \eta^{4}$ if $n \equiv 1 \bmod 6$ and zero otherwise. This implies that

$$
\mathcal{B}(\mathcal{A})=\left\{A^{n} \geq 1: n \equiv 1 \bmod 6\right\} .
$$


Since $\boldsymbol{v}(B)=v_{n}=v_{2}$ for $n \equiv 1 \bmod 6$, we obtain

$$
S(\mathcal{A} ; \Gamma(1))=\prod_{\substack{n \geq 1 \\ n \equiv 1 \bmod 6}} S_{2}\left(\Gamma(1), v_{2}\right) .
$$

(f3) Take $N=1$. So $\boldsymbol{w}(B)=w_{1}=-\frac{3}{2}$, and $n w_{1}<-2$ for $n \geq 2$. Hence $\mathcal{B}(\mathcal{A})=\{A\}$, $\boldsymbol{v}(B)=v_{1 / 2}$, and

$$
S(\mathcal{A} ; \Gamma(1))=S_{1 / 2}\left(\Gamma(1), v_{1 / 2}\right)=\mathbb{C} \eta,
$$

with $\eta(\tau)=e^{\pi i \tau / 12} \prod_{n \geq 1}\left(1-e^{2 \pi i n \tau}\right)$.

Lemma 6.2. For each $\boldsymbol{h} \in S(\mathcal{A} ; \Gamma)$, the series

$J(\boldsymbol{h} ; y, x ; t)$

$$
:=1+\sum_{n \geq 1} \sum_{B_{1}, \ldots, B_{n} \in \mathcal{B}(\mathcal{A})} R_{n}\left(\boldsymbol{h}\left(B_{1}\right), \boldsymbol{h}\left(B_{2}\right), \ldots, \boldsymbol{h}\left(B_{n}\right) ; y, x ; t\right) B_{1} B_{2} \cdots B_{n}
$$

converges and defines an element of $N(\mathcal{A})$.

Proof. The degree of $B_{1} B_{2} \cdots B_{n}$ is at least $n$. For convergence in $\boldsymbol{O}(\mathcal{A})$ there should be for each $D \geq 0$ only finitely many terms with degree at most $D$. This restricts $n$ to $n \leq D$, and the $B_{j}$ to monomials of degree bounded by $D$, of which there are only finitely many.

The terms with $n \geq 1$ cannot contribute to the constant term, hence we obtain an element of $N(\mathcal{A})$.

Remarks. (a) If $h\left(B_{i}\right)=0$ for some $i$ in the iterated integral in (6-5), then the integral vanishes. In (6-5) we could have restricted the $B_{i}$ in the sum by the condition $\boldsymbol{h}\left(B_{i}\right) \neq 0$. In particular, $J(0 ; y ; x ; t)=1$.

(b) Definition (6-5) extends definition (4-4). If $f \in S_{\mathcal{A}}(\Gamma)$ is considered as an element $\boldsymbol{h} \in S(\mathcal{A} ; \Gamma)$, as in remark (e) to Definition 6.1, then

$$
J(\boldsymbol{f} ; y, x ; t)=J(\boldsymbol{h} ; y, x ; t) .
$$

Proposition 6.3. For all $\boldsymbol{h} \in S(\mathcal{A} ; \Gamma), \gamma \in \Gamma, z, y, x \in \mathfrak{H}^{*}$,

$$
\begin{aligned}
J\left(\boldsymbol{h} ; \gamma^{-1} y, \gamma^{-1} x ; \cdot\right) & =J(\boldsymbol{h} ; y, x ; \cdot) \mid \gamma, \\
J(\boldsymbol{h} ; x, x ; t) & =1, \\
J(\boldsymbol{h} ; z, x ; t) & =J(\boldsymbol{h} ; z, y ; t) J(\boldsymbol{h} ; y, x ; t), \\
J(\boldsymbol{h} ; x, y ; t) & =J(\boldsymbol{h} ; y, x ; t)^{-1} .
\end{aligned}
$$

Proof. The relations (6-7) and (6-8) follow directly from Lemma 3.2 and (3-1). We will prove relation (6-9) in a sequence of lemmas, and finally will derive relation (6-10) from relation (6-9). 
Relation (6-9). This relation holds in a general context of iterated integrals; automorphic properties are not needed. Our proof follows [Manin 2006, Proposition 1.2] closely. We first show relation (6-9) for $x, y, z \in \mathfrak{H}$.

Lemma 6.4. For $\boldsymbol{h} \in S(\mathcal{A} ; \Gamma)$ put

$$
\Omega(\boldsymbol{h} ; z ; t):=\sum_{B \in \mathcal{B}(\mathcal{A})}(z-t)^{\boldsymbol{w}(B)} \boldsymbol{h}(B ; z) d z \cdot B .
$$

This formal series of $\boldsymbol{O}(\mathcal{A})$-valued differential forms converges, and for $z \in \mathfrak{H}$

$$
d_{z} J(\boldsymbol{h} ; z, x ; t)=\Omega(\boldsymbol{h} ; z ; t) J(\boldsymbol{h} ; z, x ; t) .
$$

Proof. The sum in (6-11) is infinite in most cases. The convergence follows from the fact that the number of monomials with a given degree is finite. The differential of a nonconstant term in (6-5) is given by

$$
\begin{aligned}
d_{z} R_{n}\left(\boldsymbol{h}\left(B_{1}\right), \boldsymbol{h}\left(B_{2}\right), \ldots, \boldsymbol{h}\left(B_{n}\right) ; z, x ; t\right) B_{1} B_{2} \cdots B_{n} \\
\quad=\boldsymbol{h}\left(B_{1} ; z\right)(z-t)^{\boldsymbol{w}\left(B_{1}\right)} B_{1} R_{n-1}\left(\boldsymbol{h}\left(B_{2}\right), \ldots, \boldsymbol{h}\left(B_{n}\right) ; z, x ; t\right) B_{2} \cdots B_{n} .
\end{aligned}
$$

With a renumbering in the summation this gives (6-12).

Lemma 6.5. $d_{z} J(\boldsymbol{h} ; z, x ; t)^{-1}=-J(\boldsymbol{h} ; z, x ; t)^{-1} \Omega(\boldsymbol{h} ; z ; t)$.

Proof. The inverse is defined by the relation

$$
1=J(\boldsymbol{h} ; z, x ; t)^{-1} J(\boldsymbol{h} ; z, x ; t) .
$$

Taking the differential of both sides gives, with (6-12),

$$
0=\left(d_{z} J(\boldsymbol{h} ; z, x ; t)^{-1}\right) J(\boldsymbol{h} ; z, x ; t)+J(\boldsymbol{h} ; z, x ; t)^{-1} \Omega(\boldsymbol{h} ; z ; t) J(\boldsymbol{h} ; z, x ; t) .
$$

Right multiplication by $J(\boldsymbol{h} ; z, x ; t)$ gives the relation in the lemma.

Lemma 6.6. For fixed $x$ and $y$, put $K(z)=J(\boldsymbol{h} ; z, y ; t)^{-1} J(\boldsymbol{h} ; z, x ; t)$. Then $K(z)=J(\boldsymbol{h} ; y, x ; t)$.

Proof. With Lemmas 6.4 and 6.5 we find

$$
\begin{aligned}
d_{z} K(z) & =-J(\boldsymbol{h} ; z, y ; t)^{-1} \Omega(\boldsymbol{h} ; z ; t) J(\boldsymbol{h} ; z, x ; t)+J(\boldsymbol{h} ; z, y ; t)^{-1} \Omega(\boldsymbol{h} ; z ; t) J(\boldsymbol{h} ; z, x ; t) \\
& =0 .
\end{aligned}
$$

Hence $K(z)$ is constant. By $(6-8)$, its value is

$$
K(y)=J(\boldsymbol{h} ; y, y ; t)^{-1} J(\boldsymbol{h}(y, x ; t)=J(\boldsymbol{h} ; y, x ; t) .
$$

Completion of the proof of relation (6-9). The relation

$$
K(z)=J(\boldsymbol{h} ; y, x ; t)=J(\boldsymbol{h} ; z, y ; t)^{-1} J(\boldsymbol{h} ; z, x ; t)
$$

implies the desired result for $z, y, x \in \mathfrak{H}$. By continuity it holds for $z, y, x \in \mathfrak{H}^{*}$. 
Relation (6-10). This relation follows from (6-8) and (6-9). This ends the proof of Proposition 6.3.

Noncommutative cocycle. From (6-7) and (6-9) it follows that for any $\boldsymbol{h} \in S(\mathcal{A} ; \Gamma)$

$$
\Psi(\boldsymbol{h})_{\gamma}:=J\left(\boldsymbol{h} ; \gamma^{-1} \infty, \infty ; t\right)
$$

defines a cocycle $\gamma \rightarrow \Psi(\boldsymbol{h})_{\gamma}$ in $Z^{1}(\Gamma ; N(\mathcal{A}))$.

If we replace $\infty$ in (6-13) by another base-point $x \in \mathfrak{H}^{*}$ we get a cocycle in the same cohomology class. So $\boldsymbol{h} \mapsto \Psi(\boldsymbol{h})$ induces a map from $S(\mathcal{A} ; \Gamma)$ to the noncommutative cohomology set $H^{1}\left(\Gamma ; N(\mathcal{A})\right.$ ), extending the map $\operatorname{Coh}_{\mathcal{A}}$ in Proposition 5.1.

The main result of this paper is the bijectivity of this map:

Theorem 6.7. Let $\mathcal{A}$ denote the choice of finitely many positive weights $w_{1}+2$, $w_{2}+2, \ldots, w_{\ell}+2$ and corresponding multiplier systems $v_{1}, \ldots, v_{\ell}$ of $\Gamma$. For each noncommutative cohomology class $c \in H^{1}(\Gamma ; N(\mathcal{A}))$ there is a unique element $\boldsymbol{h} \in S(\mathcal{A} ; \Gamma)$ such that $\Psi(\boldsymbol{h}) \in c$.

Proof. The induction runs over $k \geq 0$. We start with a cocycle $\mathrm{X}^{0} \in Z^{1}(\Gamma ; N(\mathcal{A}))$, and replace it in the course of an induction procedure by cocycles $X^{1}, X^{2}, \ldots$ in the same cohomology class. During the induction we form a sequence $\boldsymbol{h}_{0}, \boldsymbol{h}_{1}, \ldots, \boldsymbol{h}_{k}, \ldots$ of elements of $S(\mathcal{A} ; \Gamma)$, and a strictly increasing sequence of integers $c_{0}, c_{1}, \ldots$ The connection between the induction quantities $X^{k}, \boldsymbol{h}_{k}$ and $c_{k}$ is given by the requirement that at each stage of the induction the following conditions hold:

(H) $\boldsymbol{h}_{k}(B)=0$ for all $B$ with $d(B)>c_{k}$.

(XPs) If $X_{\gamma}^{k}-\Psi\left(\boldsymbol{h}_{k}\right)_{\gamma}=\sum_{B} a(\gamma, B) B$, where the sum $B$ runs over all noncommutative polynomials in $A_{1}, \ldots, A_{\ell}$, then for each $\gamma \in \Gamma$

$$
a(\gamma, B)=0 \quad \text { for all } B \text { with } d(B) \leq k .
$$

Either at a certain stage $k$ in the induction procedure the process stops, and we take $\boldsymbol{h}=\boldsymbol{h}_{k}$, or the process goes on indefinitely, in which case we construct $\boldsymbol{h}$ as a limit of the $\boldsymbol{h}_{k}$. In both cases we show that $\Psi(\boldsymbol{h})$ is in the cohomology class of the $X^{k}$, and that the element $\boldsymbol{h}$ is uniquely determined.

Start of the induction. For a given cocycle $\mathrm{X}^{0}$ in the cohomology class $c$ we put $\boldsymbol{h}_{0}=0$ and $c_{k}=0$. Then for all $\gamma \in \Gamma$

$$
\mathrm{X}_{\gamma}^{0}-\Psi\left(\boldsymbol{h}_{0}\right)_{\gamma}=\mathrm{X}_{\gamma}^{0}-1
$$

has no constant term, and conditions (H) and (XPs) are trivially satisfied.

Has the end of the induction process been reached? If $\mathrm{X}^{k}=\Psi\left(\boldsymbol{h}_{k}\right)$, we have found a description of the class $c$ as required in the theorem. This may happen already at the start of the induction if $X^{0}$ is the trivial cocycle $\gamma \mapsto 1$. 
Induction, choice of $c_{k+1}$. If the process has not ended, then the difference $Y^{k}:=$ $\mathrm{X}^{k}-\Psi\left(\boldsymbol{h}_{k}\right)$ determines a nonzero map $\gamma \mapsto Y_{\gamma}^{k}$ from $\Gamma$ to $\boldsymbol{O}(\mathcal{A})$. It is not a cocycle.

We define $c_{k+1}$ as the minimum degree such that $Y_{\gamma}^{k} \in \boldsymbol{O}(\mathcal{A})$ has nonzero terms of degree $c_{k+1}$ in $A_{1}, \ldots, A_{\ell}$ for some $\gamma \in \Gamma$. Since condition (XPs) holds for $k$ we have $c_{k+1}>c_{k}$.

Cocycle relation. The cocycle relations for the noncommutative cocycles $\mathrm{X}^{k}$ and $\Psi\left(\boldsymbol{h}_{k}\right)$ give

$$
\begin{aligned}
\mathrm{Y}_{\gamma \delta}^{k} & =\left(\left(\mathrm{Y}_{\gamma}^{k}+\Psi\left(\boldsymbol{h}_{k}\right)_{\gamma}\right) \mid \delta\right)\left(\mathrm{Y}_{\delta}^{k}+\Psi\left(\boldsymbol{h}_{k}\right)_{\delta}\right)-\left(\Psi\left(\boldsymbol{h}_{k}\right)_{\gamma} \mid \delta\right) \Psi\left(\boldsymbol{h}_{k}\right)_{\delta} \\
& =\left(\mathrm{Y}_{\gamma}^{k} \mid \delta\right) \Psi\left(\boldsymbol{h}_{k}\right)_{\delta}+\left(\Psi\left(\boldsymbol{h}_{k}\right)_{\gamma} \mid \delta\right) \mathrm{Y}_{\delta}^{k}+\left(\mathrm{Y}_{\gamma}^{k} \mid \delta\right) \mathrm{Y}_{\delta}^{k} .
\end{aligned}
$$

By condition (XPs) and the choice of $c_{k+1}$, the element $Y_{\gamma}^{k} \in \boldsymbol{O}(\mathcal{A})$ has no terms with degree less than $c_{k+1}$. We denote by $\bar{Y}_{\gamma}^{k}$ the sum of the terms of $Y_{\gamma}^{k}$ with exact degree $c_{k+1}$. We consider relation (6-14) modulo terms with degree strictly larger than $c_{k+1}$ :

$$
\overline{\mathrm{Y}}_{\gamma \delta}^{k} \equiv\left(\overline{\mathrm{Y}}_{\gamma}^{k} \mid \delta\right) \Psi\left(\boldsymbol{h}_{k}\right)_{\delta}+\left(\Psi\left(\boldsymbol{h}_{k}\right)_{\gamma} \mid \delta\right) \overline{\mathrm{Y}}_{\delta}^{k}+0 .
$$

In the two products only the constant term 1 of $\Psi\left(\boldsymbol{h}_{k}\right)_{\gamma}$ and $\Psi\left(\boldsymbol{h}_{k}\right)_{\delta}$ is relevant, and we obtain

$$
\overline{\mathrm{Y}}_{\gamma \delta}^{k}=\overline{\mathrm{Y}}_{\gamma}^{k} \mid \delta+\overline{\mathrm{Y}}_{\delta}^{k}
$$

So the noncommutative cocycle relations for $\mathrm{X}^{k}$ and $\Psi\left(\boldsymbol{h}_{k}\right)$ imply that $\gamma \mapsto \overline{\mathrm{Y}}_{\gamma}^{k}$ is a commutative cocycle with values in the additive group of $\boldsymbol{O}(\mathcal{A})$.

The elements $\bar{Y}_{\gamma}^{k}$ have the form

$$
\overline{\mathrm{Y}}_{\gamma}^{k}=\sum_{n=1}^{K} \varphi_{\gamma}^{n} C_{n}, \quad C_{n}=A_{p_{n, 1}} A_{p_{n, 2}} \cdots A_{p_{n, c_{k+1}}},
$$

with $\varphi_{\gamma}^{n} \in V\left(\boldsymbol{v}\left(C_{n}\right), \boldsymbol{w}\left(C_{n}\right)\right)$. The $C_{n}$ have degree $c_{k+1}$ in $A_{1}, \ldots, A_{\ell}$. For each $n$ there is some $\gamma \in \Gamma$ for which $\varphi_{\gamma}^{n} \neq 0$. Relation (6-16) implies that each component of $\bar{Y}^{k}$ is a cocycle: $\varphi^{n} \in Z^{1}\left(\Gamma ; V\left(v\left(C_{n}\right), \boldsymbol{w}\left(C_{n}\right)\right)\right)$. By Theorem 2.1 there exist $a_{n} \in V\left(\boldsymbol{v}\left(C_{n}\right), \boldsymbol{w}\left(C_{n}\right)\right)$ and unique cusp forms $g_{n} \in S_{\boldsymbol{w}\left(C_{n}\right)+2}\left(\Gamma, \boldsymbol{v}\left(C_{n}\right)\right)$ such that for all $\gamma \in \Gamma$

$$
\varphi_{\gamma}^{n}=-\psi_{g_{n}, \gamma}+\left.a_{n}\right|_{\boldsymbol{v}\left(C_{n}\right),-w\left(C_{n}\right)}(\gamma-1) .
$$

Induction, choice of $\mathrm{X}^{k+1}$. Take

$$
H_{k}=1-\sum_{n=1}^{K} a_{n} C_{n}
$$

This is an element of $N(\mathcal{A})$. We define the cocycle $\mathrm{X}^{k+1}$ in the same class as $\mathrm{X}^{k}$ by

$$
\mathrm{X}_{\gamma}^{k+1}=\left(H_{k} \mid \gamma\right) \mathrm{X}_{\gamma}^{k} H_{k}^{-1} \text {. }
$$


Induction, choice of $\boldsymbol{h}_{k+1}$. It may happen that $C_{n} \notin \mathcal{B}(\mathcal{A})$ for some $n \in\{1, \ldots, k\}$. Then $S_{\boldsymbol{w}\left(C_{n}\right)+2}\left(\Gamma, \boldsymbol{v}\left(C_{n}\right)\right)=0$, and $\varphi^{n}$ is a coboundary and $g_{n}=0$.

By condition (H) we have $\boldsymbol{h}_{k}\left(C_{n}\right)=0$ for $1 \leq n \leq K$. We construct $\boldsymbol{h}_{k+1}$ from $\boldsymbol{h}_{k}$ by taking $\boldsymbol{h}_{k+1}\left(C_{n}\right)=g_{n}$ for those $n$ for which $C_{n} \in \mathcal{B}(\mathcal{A})$, and $\boldsymbol{h}_{k+1}(B)=\boldsymbol{h}_{k}(B)$ otherwise. So $\boldsymbol{h}_{k}(B)=\boldsymbol{h}_{k+1}(B)$ for all $B$ with $d(B)>c_{k+1}$, and condition $(\mathrm{H})$ stays valid for $k+1$. If $C_{n} \notin \mathcal{B}(\mathcal{A})$ for all $n$, then $\boldsymbol{h}_{k+1}=\boldsymbol{h}_{k}$.

Induction, check of condition (XPs) for $k+1$. Modulo terms of order larger than $c_{k+1}$, we have

$$
\begin{aligned}
\mathrm{X}_{\gamma}^{k+1} & \equiv\left(1-\sum_{n} a_{n} \mid \gamma C_{n}\right)\left(\Psi\left(\boldsymbol{h}_{k}\right)_{\gamma}+\overline{\mathrm{Y}}_{\gamma}^{k}\right)\left(1+\sum_{n} a_{n} C_{n}\right) \\
& \equiv \Psi\left(\boldsymbol{h}_{k}\right)_{\gamma}+\bar{Y}_{\gamma}^{k}-\sum_{n} a_{n} \mid \gamma C_{n}+\sum_{n} a_{n} C_{n} \\
& \equiv \Psi\left(\boldsymbol{h}_{k}\right)_{\gamma}+\sum_{n}\left(-\psi_{g_{n}, \gamma}+a_{n}\left|(\gamma-1)-a_{n}\right| \gamma+a_{n}\right) C_{n} \\
& =\Psi\left(\boldsymbol{h}_{k}\right)_{\gamma}+\sum_{n} R_{1}\left(g_{n} ; \gamma^{-1} \infty, \infty\right) C_{n}
\end{aligned}
$$

By (6-13) and (6-5), we have

$\Psi\left(\boldsymbol{h}_{k+1}\right)_{\gamma}$

$$
\begin{array}{r}
=1+\sum_{m \geq 1} \sum_{B_{1}, \ldots, B_{m} \in \mathcal{B}(\mathcal{A})} R_{m}\left(\boldsymbol{h}_{k+1}\left(B_{1}\right), \boldsymbol{h}_{k+1}\left(B_{2}\right), \ldots, \boldsymbol{h}_{k+1}\left(B_{m}\right) ; \gamma^{-1} \infty, \infty ; t\right) \\
\times B_{1} B_{2} \cdots B_{m}
\end{array}
$$

in which we can leave out the terms in which a $B_{i}$ occurs with $d\left(B_{i}\right)>c_{k+1}$, by condition $(\mathrm{H})$. If we leave out the terms with a $B_{i}$ for which $d\left(B_{i}\right)>c_{k}$, we obtain $\Psi\left(\boldsymbol{h}_{k}\right)_{\gamma}$. If there is a $B_{i}$ with $d\left(B_{i}\right)>c_{k}$ this is one of the $C_{n}$ in (6-17), with $d\left(B_{i}\right)=d\left(C_{n}\right)=c_{k+1}$. Working modulo terms with degree larger than $c_{k+1}$ we obtain

$$
\begin{aligned}
\Psi\left(\boldsymbol{h}_{k+1}\right)_{\gamma}-\Psi\left(\boldsymbol{h}_{k}\right)_{\gamma} & \equiv \sum_{\substack{1 \leq n \leq K \\
C_{n} \in \mathcal{B}(\mathcal{A})}} R_{1}\left(\boldsymbol{h}_{k+1}\left(C_{n}\right) ; \gamma^{-1} \infty, \infty ; t\right) C_{n} \\
& =\sum_{\substack{1 \leq n \leq K \\
C_{n} \in \mathcal{B}(\mathcal{A})}} R_{1}\left(g_{n} ; \gamma^{-1} \infty, \infty ; t\right) C_{n} .
\end{aligned}
$$

A comparison of (6-21) and (6-22) gives condition (XPs) for $k+1$.

The induction may halt. It may happen that the induction stops at stage $k$; namely, if $X^{k}=\Psi\left(\boldsymbol{h}_{k}\right)$. Then we have found an element $\boldsymbol{h}=\boldsymbol{h}_{k} \in S(\mathcal{A} ; \Gamma)$ such that $\Psi(\boldsymbol{h})$ is in the class $c$. 
The induction may have infinitely many steps. It may also happen that we have obtained after infinitely many steps an infinite sequence of cocycles $X^{k}$ in the class $c$, an infinite sequence of $\boldsymbol{h}_{k}$, and a strictly increasing sequence of $c_{k}$ satisfying conditions (H) and (XPs) for all $k$. For each monomial $B \in \mathcal{B}(\mathcal{A})$ there is at most one $k$ such that $h_{n}(B)=0$ for $n \leq k$, and $h_{n}(B)=h_{k+1}(B)$ for $n \geq k+1$. So the componentwise limit $\boldsymbol{h}:=\lim _{k \rightarrow \infty} \boldsymbol{h}_{k}$ exists.

The construction of the sequence $\left(X^{k}\right)_{k}$ implies that

$$
\mathrm{X}_{\gamma}^{k}=\left(\left(H_{k-1} H_{k-2} \cdots H_{0}\right) \mid \gamma\right) \mathrm{X}_{\gamma}^{0}\left(H_{k-1} H_{k-2} \cdots H_{0}\right)^{-1},
$$

with $H_{k}$ as in (6-19). The infinite product $H=\cdots H_{2} H_{1} H_{0}$ converges in $N(\mathcal{A})$, since each $H_{k}$ equals 1 plus a term in degree $c_{k+1}$. Similarly, $J\left(\boldsymbol{h} ; \gamma^{-1} \infty, \infty ; t\right)$ is the limit of the $J\left(\boldsymbol{h}_{k} ; \gamma^{-1} \infty, \infty ; t\right)$ as $k \rightarrow \infty$, since enlarging $k$ we change only terms of degrees larger than $c_{k}$. Condition (XPs) is valid for all $k$, so the conclusion is that, in the limit,

$$
(H \mid \gamma) \mathrm{X}^{0} H^{-1}=\Psi(\boldsymbol{h}) .
$$

Uniqueness. Let $\mathrm{X}^{0}=\Psi\left(\boldsymbol{h}^{\prime}\right)$ for some $\boldsymbol{h}^{\prime} \in S(\mathcal{A} ; \Gamma)$. We claim that, in the induction procedure described above applied to this cocycle $X^{0}$, we have at each stage

$$
\begin{aligned}
\boldsymbol{h}_{k}(B) & =\boldsymbol{h}^{\prime}(B) & & \text { for all } B \in \mathcal{B}(\mathcal{A}) \text { with } d(B) \leq c_{k} ; \\
\mathrm{X}^{k} & \equiv \Psi\left(\boldsymbol{h}^{\prime}\right) & & \text { modulo terms of degree larger than } c_{k} .
\end{aligned}
$$

This is true at the start of the induction (use $c_{0}=0$ ).

At stage $k$, the nonzero terms with lowest degree in

$$
Y_{\gamma}^{k}=J\left(\boldsymbol{h}^{\prime} ; \gamma^{-1} \infty, \infty ; t\right)-J\left(\boldsymbol{h}_{k} ; \gamma^{-1} \infty, \infty ; t\right)
$$

are due to the $C \in \mathcal{B}(\mathcal{A})$ with degree equal to $c_{k+1}$. So

$$
\bar{Y}_{\gamma}=\sum_{\substack{C \in \mathcal{B}(\mathcal{A}) \\ d(C)=c_{k+1}}} R_{1}\left(\boldsymbol{h}^{\prime}(B) ; \gamma^{-1} \infty, \infty ; t\right) C .
$$

Let us number the monomials in this sum as $C_{1}, \ldots, C_{K}$. Then $\varphi_{\gamma}^{n}$ in (6-18) is equal to $-\psi_{\boldsymbol{h}^{\prime}\left(C_{n}\right), \gamma}$, and $a_{n}=0, H_{k}=1$. This implies that $\boldsymbol{h}_{k+1}(C)=\boldsymbol{h}^{\prime}(C)$ for the monomials $C \in \mathcal{B}(\mathcal{A})$ with degree $c_{k+1}$, and $\mathrm{X}^{k+1}=\mathrm{X}^{k} \equiv \Psi\left(\boldsymbol{h}^{\prime}\right)$ modulo terms with degree larger than $c_{k+1}$.

At the end of the induction process we have $\mathcal{B}=\mathcal{B}^{\prime}$, thus obtaining uniqueness.

Concluding remarks. (a) Manin [2005; 2006] used formal series similar to those in (4-4) to get a simple description of relations among iterated integrals. In that approach the noncommutative cohomology set $H^{1}(\Gamma ; N(\mathcal{A}))$ is a tool. In this paper we further study the cohomology set $H^{1}(\Gamma ; N(\mathcal{A}))$. 
(b) One may apply the approach of this paper to weights in $\mathbb{Z}_{\geq 2}$ and trivial multiplier systems. Then the iterated integrals are polynomial functions. These are in a much smaller $\Gamma$-module than the functions with polynomial growth that we employ. The consequence is that the theorem analogous to the theorem of Knopp and Mawi (Theorem 2.1) does not hold. Cocycles attached to conjugates of holomorphic cusp forms have to be considered as well (see [Knopp 1974]). However, iterated integrals in which occur both holomorphic and antiholomorphic cusp forms satisfy more complicated decomposition relations. We think that Manin's formalism does not work in that situation.

(c) The same problem occurs if we use the modules in Theorems B and D of [Bruggeman et al. 2014], unless we pick the weights $w_{j}+2$ in such a way that the elements $\boldsymbol{w}(C)$ that occur in the sums defining $J(\mathcal{B} ; y, x ; t)$ are never in $\mathbb{Z}_{\geq 0}$.

(d) We work with iterated integrals of the type in (3-1). Equation (1-1) defines iterated integrals depending on variables $t_{1}, \ldots, t_{\ell}$ all running independently through the lower half-plane. It would be nice to have results for the corresponding noncommutative cocycles. These cocycles can be defined, and one can show injectivity of the map from cusp forms to cohomology, like in Proposition 5.1. We did not manage to adapt the proof of Theorem 6.7 to cocycles of this type. The problem is to construct formal sequences of the type in (6-5) such that they have the lowest degree terms in a prescribed summand in the decomposition of the tensor products $V\left(v_{1}, w_{1}\right) \otimes \cdots \otimes V\left(v_{\ell}, w_{\ell}\right)$ into submodules.

\section{Acknowledgement}

The authors would like to thank the referees for numerous helpful comments and suggestions which greatly improved the exposition of this paper, in particular the introduction.

\section{References}

[Brown 2014] F. Brown, "Motivic periods and $\mathbb{P}^{1} \backslash\{0,1, \infty\}$ ", preprint, 2014. arXiv 1407.5165

[Bruggeman et al. 2014] R. Bruggeman, Y. Choie, and N. Diamantis, "Holomorphic automorphic forms and cohomology", preprint, 2014. arXiv 1404.6718

[Chen 1977] K. T. Chen, "Iterated path integrals", Bull. Amer. Math. Soc. 83:5 (1977), 831-879. MR 0454968 Zbl 0389.58001

[Choie 2014] Y. Choie, "Parabolic cohomology and multiple Hecke $L$-values", preprint, Pohang University of Science and Technology, 2014, Available at http://math.postech.ac.kr/ yjc/para-Choie.pdf.

[Hain 2015] R. Hain, “The Hodge-de Rham theory of modular groups", preprint, 2015. arXiv 1403. 6443

[Knopp 1974] M. I. Knopp, "Some new results on the Eichler cohomology of automorphic forms", Bull. Amer. Math. Soc. 80 (1974), 607-632. MR 0344454 Zbl 0292.10022 
[Knopp and Mawi 2010] M. Knopp and H. Mawi, "Eichler cohomology theorem for automorphic forms of small weights", Proc. Amer. Math. Soc. 138:2 (2010), 395-404. MR 2557156 Zbl 1233.11058

[Manin 2005] Y. I. Manin, "Iterated Shimura integrals", Mosc. Math. J. 5:4 (2005), 869-881, 973. MR 2266463 Zbl 1215.11052

[Manin 2006] Y. I. Manin, "Iterated integrals of modular forms and noncommutative modular symbols", pp. 565-597 in Algebraic geometry and number theory, edited by V. Ginzburg, Progr. Math. 253, Birkhäuser, Boston, 2006. MR 2263200 Zbl 1184.11019

[Provost 2014] N. Provost, Valeurs multiples de fonctions L de formes modulaires, thèse de doctorat, Université Paris Diderot, 2014. arXiv 1604.01913

Communicated by Yuri Manin

Received 2015-06-29 Revised 2016-02-26 Accepted 2016-02-28

r.w.bruggeman@uu.nl

Mathematisch Instituut, Universiteit te Utrecht, Postbus 80010, 3508 TA Utrecht, Netherlands

yjc@postech.ac.kr

Department of Mathematics and PMI, Postech, Pohang 790-784, South Korea 


\section{Algebra \& Number Theory}

msp.org/ant

\section{EDITORS}

MANAGING EDITOR

Bjorn Poonen

Massachusetts Institute of Technology

Cambridge, USA

\author{
EDITORIAL BOARD CHAIR \\ David Eisenbud \\ University of California \\ Berkeley, USA
}

\section{BOARD OF EDITORS}

Georgia Benkart

Dave Benson

Richard E. Borcherds

John H. Coates

J-L. Colliot-Thélène

Brian D. Conrad

Hélène Esnault

Hubert Flenner

Sergey Fomin

Edward Frenkel

Andrew Granville

Joseph Gubeladze

Roger Heath-Brown

Craig Huneke

Kiran S. Kedlaya

János Kollár

Yuri Manin

Philippe Michel
University of Wisconsin, Madison, USA

University of Aberdeen, Scotland

University of California, Berkeley, USA

University of Cambridge, UK

CNRS, Université Paris-Sud, France

Stanford University, USA

Freie Universität Berlin, Germany

Ruhr-Universität, Germany

University of Michigan, USA

University of California, Berkeley, USA

Université de Montréal, Canada

San Francisco State University, USA

Oxford University, UK

University of Virginia, USA

Univ. of California, San Diego, USA

Princeton University, USA

Northwestern University, USA

École Polytechnique Fédérale de Lausanne
Susan Montgomery

Shigefumi Mori

Raman Parimala

Jonathan Pila

Anand Pillay

Victor Reiner

Peter Sarnak

Joseph H. Silverman

Michael Singer

Vasudevan Srinivas

J. Toby Stafford

Ravi Vakil

Michel van den Bergh

Marie-France Vignéras

Kei-Ichi Watanabe

Efim Zelmanov

Shou-Wu Zhang
University of Southern California, USA

RIMS, Kyoto University, Japan

Emory University, USA

University of Oxford, UK

University of Notre Dame, USA

University of Minnesota, USA

Princeton University, USA

Brown University, USA

North Carolina State University, USA

Tata Inst. of Fund. Research, India

University of Michigan, USA

Stanford University, USA

Hasselt University, Belgium

Université Paris VII, France

Nihon University, Japan

University of California, San Diego, USA

Princeton University, USA

\section{PRODUCTION}

production@msp.org

Silvio Levy, Scientific Editor

See inside back cover or msp.org/ant for submission instructions.

The subscription price for 2016 is US \$/year for the electronic version, and \$/year (+\$, if shipping outside the US) for print and electronic. Subscriptions, requests for back issues and changes of subscribers address should be sent to MSP.

Algebra \& Number Theory (ISSN 1944-7833 electronic, 1937-0652 printed) at Mathematical Sciences Publishers, 798 Evans Hall \#3840, c/o University of California, Berkeley, CA 94720-3840 is published continuously online. Periodical rate postage paid at Berkeley, CA 94704, and additional mailing offices.

ANT peer review and production are managed by EditFLOW ${ }^{\circledR}$ from MSP.

\section{PUBLISHED BY}

mathematical sciences publishers

nonprofit scientific publishing

http://msp.org/

(C) 2016 Mathematical Sciences Publishers 


\section{Algebra \& Number Theory}

Volume $10 \quad$ No. $3 \quad 2016$

Group schemes and local densities of ramified hermitian lattices in residue characteristic 2: Part I

SUNGMUN CHO

Presentation of affine Kac-Moody groups over rings

DANIEL ALLCOCK

Discriminant formulas and applications

Kenneth Chan, Alexander A. Young and James J. Zhang

LUIS E. GARCIA

Multiple period integrals and cohomology

RoElof W. BRUGGEMAN and YoungJu CHOIE

The existential theory of equicharacteristic henselian valued fields

SYLVY ANSCOMBE and ARNO FEHM

SOMNATH JHA, TADASHI OCHIAI and GERGELY ZÁBRÁDI 